\title{
Ionic liquids derived from pyridine and their application in Suzuki-Miyaura reaction under microwave irradiation
}

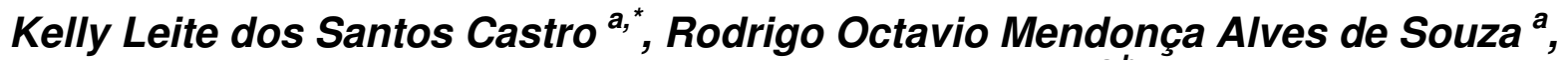 Leandro Soter de Mariz e Miranda ${ }^{a, b}$. \\ ${ }^{a}$ Grupo de Biocatálise e Síntese Orgânica, Instituto de Química, Universidade Federal do Rio de Janeiro,}

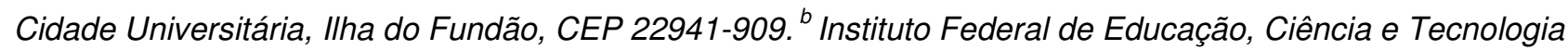
do Rio de Janeiro, Maracanã, Rua Senador Furtado, 121, Rio de Janeiro, CEP 20270-021.

*kellylsc@ig.com.br

Keywords: Suzuki-Miyaura reaction, ionic liquid, microwave in organic synthesis

\section{INTRODUCTION}

The Suzuki-Miyaura reaction, the palladium catalyzed coupling of organic halides and organic compounds of boron, is one of the most efficient alternatives to the formation of carbon-carbon bonds, with success in the synthesis of biaryls. ${ }^{1}$ Consequently, the interest in creating systems that are efficient for this reaction is an area of intense research efforts.

Ionic Liquids have been increasingly studied, as an environmentally acceptable alternative to replace the use of organic solvents. These substances have an important advantage that comes from their ionic characteristics such as high density and low vapor pressure. ${ }^{2}$ In this paper we wish to present the results of the Suzuki-Miyaura coupling reaction promoted by microwave irradiation (MW) using ionic liquids derived from different pyridinium cations (Fig. 1 ), assisted by microwave irradiation (MW).

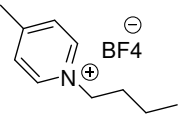

$[4-\mathrm{MBpi}]\left[\mathrm{BF}_{4}\right]$

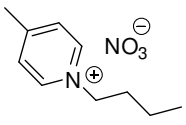

$[4-\mathrm{MBpi}]\left[\mathrm{NO}_{3}\right]$

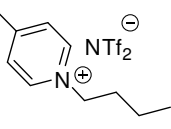

[4-MBpi][NTF 2$]$

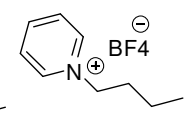

$[\mathrm{Bpi}]\left[\mathrm{BF}_{4}\right]$
Figure 1. Pyridinium lonic liquid derivatives.

\section{RESULTS AND DISCUSSION}

The study of Suzuki- Miyaura reaction was initiated by the reaction between phenylboronic acid and 4bromo-acetophenone using a palladium catalyst and triethylamine as a base under microwave irradiation, figure 2. To perform the reaction two catalysts were chosen: $\mathrm{Pd}(\mathrm{OAc})_{2}$ (3 mol\%) and $\mathrm{Pd} / \mathrm{Nb}_{2} \mathrm{O}_{5} \quad(3 \%$ $\mathrm{MW})$.

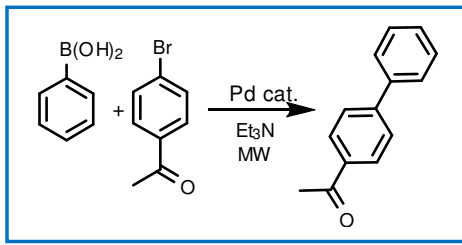

Figure 2. The Suzuki- Miyaura reaction
Table 1. Results on the Suzuki-Miyaura reaction

\begin{tabular}{|c|c|c|c|}
\hline Entry & Solvent & Catalyst & Conv. $(\%)^{a}$ \\
\hline $1^{b}$ & [4-MBpy][BF & $\mathrm{Pd}(\mathrm{OAc})_{2}$ & 30 \\
\hline $2^{b}$ & $\mathrm{H}_{2} \mathrm{O}$ & $\mathrm{Pd}(\mathrm{OAc})_{2}$ & 68 \\
\hline $3^{b}$ & $\begin{array}{c}{[4-\mathrm{MBpy}]\left[\mathrm{BF}_{4}\right] / \mathrm{H}_{2} \mathrm{O}} \\
(1: 1)\end{array}$ & $\mathrm{Pd}(\mathrm{OAc})_{2}$ & 94 \\
\hline $4^{b}$ & $\begin{array}{l}{[4-\mathrm{MBpy}]\left[\mathrm{BF}_{4}\right] /} \\
\mathrm{H}_{2} \mathrm{O}(0.75: 0.25)\end{array}$ & $\mathrm{Pd}(\mathrm{OAc})_{2}$ & 97 \\
\hline $5^{b}$ & $\begin{array}{c}{[4-\mathrm{MBpy}]\left[\mathrm{BF}_{4}\right] / \mathrm{H}_{2} \mathrm{O}} \\
(0.85: 0.15)\end{array}$ & $\mathrm{Pd}(\mathrm{OAc})_{2}$ & 93 \\
\hline $6^{c}$ & $\begin{array}{c}\text { [4-MBpy] }\left[\mathrm{BF}_{4}\right] / \mathrm{H}_{2} \mathrm{O} \\
(1: 1)\end{array}$ & $\mathrm{Pd} / \mathrm{Nb}_{2} \mathrm{O}_{5}$ & 77 \\
\hline $7^{\mathrm{c}}$ & $\begin{array}{c}{[4-\mathrm{MBpy}]\left[\mathrm{BF}_{4}\right] / \mathrm{H}_{2} \mathrm{O}} \\
(1: 1)\end{array}$ & $\mathrm{Pd} / \mathrm{Nb}_{2} \mathrm{O}_{5}$ & 89 \\
\hline $8^{b}$ & {$[4-\mathrm{MBpy}]\left[\mathrm{NO}_{3}\right](1: 1)$} & $\mathrm{Pd}(\mathrm{OAc})_{2}$ & 21 \\
\hline $9^{b}$ & [4-MBpy][NTF 2$](1: 1)$ & $\mathrm{Pd}(\mathrm{OAc})_{2}$ & 74 \\
\hline $10^{b}$ & {$[\mathrm{Bpy}]\left[\mathrm{BF}_{4}\right](1: 1)$} & $\mathrm{Pd}(\mathrm{OAc})_{2}$ & 92 \\
\hline
\end{tabular}

According to the Table 1, better results were observed when [4-MBpy][BF4]/H2O and $\mathrm{Pd}(\mathrm{OAc}) 2$ were used (Table 1, entries 3,4 and 5), the best results come from the combination $\mathrm{LI} / \mathrm{H}_{2} \mathrm{O}$, entries 3 , 4 and 5 . Supported catalyst also resulted in good yields, although at higher reaction times, entries 6 and 7.

\section{CONCLUSION}

It can be concluded that the use of environmentally sound conditions [4-MBpy] $\left[\mathrm{BF}_{4}\right]$ in conjunction with $\mathrm{H}_{2} \mathrm{O}$ as solvent in the Suzuki-Miyaura reaction, led to a reaction with very high yields and short reaction times under microwave irradiation.

\section{ACKNOWLEDGEMENTS}

\section{CAPES}

\section{REFERENCES}

Miyaura, N..; Yanigi, T.; Suzuki, A. Synth. Commun.. 1981, 11, 513.

2 Silva, M. F.; Lacerda, B. S. P.; Junior, J. J. Química Nova, 2005, 28, 103. 